\title{
Testicular Mass
}

National Cancer Institute

\section{Source}

National Cancer Institute. Testicular Mass. NCI Thesaurus. Code C61394.

An abnormal growth in the testis. 